\title{
Síndrome de Vasoconstrição Cerebral Reversível: uma Causa Importante de Acidentes Vasculares Cerebrais no Puerpério
}

\author{
Reversible Cerebral Vasoconstriction Syndrome: an Important Cause of \\ Stroke in the Puerperium
} Tiago RODRIGUES ${ }^{1}$, Rui LOUREIRO², Raquel SAMÕES ${ }^{2}$, Viriato ALVES ${ }^{1}$, Cristina RAMOS ${ }^{1}$, Carlos CORREIA²
Acta Med Port 2014 Jul-Aug;27(4):515-518

\section{RESUMO}

A síndrome de vasoconstrição cerebral reversível é uma doença cerebrovascular rara caracterizada por vasoconstrição segmentar das artérias cerebrais, mais frequentemente espontaneamente reversível. Pode ocorrer no período pós-parto, manifestando-se através de sintomas neurológicos agudos, sendo que os exames de imagem desempenham um papel fundamental para o seu diagnóstico. Embora classicamente considerada uma doença autolimitada e benigna, pode apresentar cursos menos favoráveis com importante morbilidade e mortalidade associada. Descrevemos um caso de síndrome de vasoconstrição cerebral reversível no puerpério, com vasospasmo cerebral progressivo causando lesões vasculares cerebrais isquémicas e hemorrágicas. Pretendemos deste modo chamar a atenção para as potenciais complicações desta entidade que implicam uma vigilância clínica e imagiológica intensa.

Palavras-chave: Acidente Vascular Cerebral; Artérias Cerebrais; Puerpério; Vasoconstrição; Vasospasmo Intracraniano.

\section{ABSTRACT}

Reversible cerebral vasoconstriction syndrome is a rare cerebrovascular disorder characterized by cerebral arterial segmental vasoconstriction, usually spontaneously reversible. This disease can occur in the postpartum period, manifesting itself through acute neurologic symptoms, and the imaging studies play a fundamental role in its diagnosis. Although classically considered a benign and self-limiting disease, it may present less favorable courses with significant associated morbidity and mortality. We describe a case of reversible cerebral vasoconstriction syndrome in the puerperium, with progressive cerebral vasospasm causing ischemic and hemorrhagic strokes. We intend to make an alert to the potential complications of this entity that demand a close clinical and imagiological monitoring.

Keywords: Stroke; Cerebral Arteries; Puerperal Disorders; Vasoconstriction; Vasospasm, Intracranial.

\section{INTRODUÇÂO}

A síndrome de vasoconstrição cerebral reversível (SVCR) compreende um grupo de entidades caraterizadas por vasoconstrição segmentar multifocal das artérias intracranianas. ${ }^{1} \mathrm{~A}$ sua ocorrência no puerpério, anteriormente conhecida como angiopatia pós-parto, ${ }^{2,3}$ é rara, ${ }^{4,5}$ manifestando-se precocemente no período pós-parto ${ }^{4-6}$ através de sintomas neurológicos agudos, nomeadamente cefaleias, crises convulsivas, acidentes vasculares cerebrais (isquémicos e hemorrágicos) e/ou encefalopatia..-7 Os exames imagiológicos, em particular os estudos angiográficos, desempenham um papel fundamental para o seu diagnóstico ao demonstrarem as referidas estenoses arteriais multifocais. Permitem ainda identificar as potenciais complicações decorrentes do vasospasmo arterial, designadamente hemorragias intraparenquimatosas ou subaracnoideias, enfartes e/ou edema cerebral. ${ }^{4,6}$

$\mathrm{O}$ seu processo fisiopatológico permanece ainda mal compreendido. No estudo anatomopatológico de artérias cerebrais envolvidas em casos fatais, foram apenas observadas alterações focais mínimas na parede arterial, favorecendo uma vasoconstrição funcional em detrimento de um processo inflamatório/vasculítico. ${ }^{7}$ Alguns dos casos que ocorrem no puerpério são considerados iatrogénicos, mais comummente associados à administração de bromocripti- na, ${ }^{6}$ mas também ao uso de simpaticomiméticos, triptanos e inibidores seletivos da recaptação da serotonina. ${ }^{3}$

A vasoconstrição arterial é mais frequentemente auto-limitada e espontaneamente reversível, sendo o prognóstico classicamente considerado favorável. 4,7 Contudo, alguns casos podem ser fatais, ${ }^{7}$ e numa série de 18 casos de SVCR em puérperas publicada recentemente os autores verificaram que metade das doentes permaneceu com défices neurológicos residuais $(28 \%)$ ou faleceram $(22 \%){ }^{4}$

\section{CASO CLÍNICO}

Puérpera de 36 anos de idade, com antecedentes pessoais de enxaqueca com aura, aplasia medular adquirida não imune, um abortamento espontâneo no primeiro trimestre e história familiar de aneurisma cerebral (na mãe).

A gestação foi complicada por anemia detetada no primeiro trimestre, tendo sido inicialmente tratada com eritropoietina e suplementação vitamínica mas com necessidade de suporte transfusional no segundo trimestre da gestação. No final da gravidez, apresentou um quadro de anasarca, sem albuminúria e com valores de tensão arterial normais, não satisfazendo deste modo os critérios de pré-eclampsia/eclampsia.

No terceiro dia após parto distócito (cesariana sob

1. Serviço de Neurorradiologia. Centro Hospitalar do Porto. Porto. Portugal.

2. Serviço de Neurologia. Centro Hospitalar do Porto. Porto. Portugal.

Recebido: 20 de Outubro de 2013 - Aceite: 09 de Janeiro de 2014 | Copyright @ Ordem dos Médicos 2014 


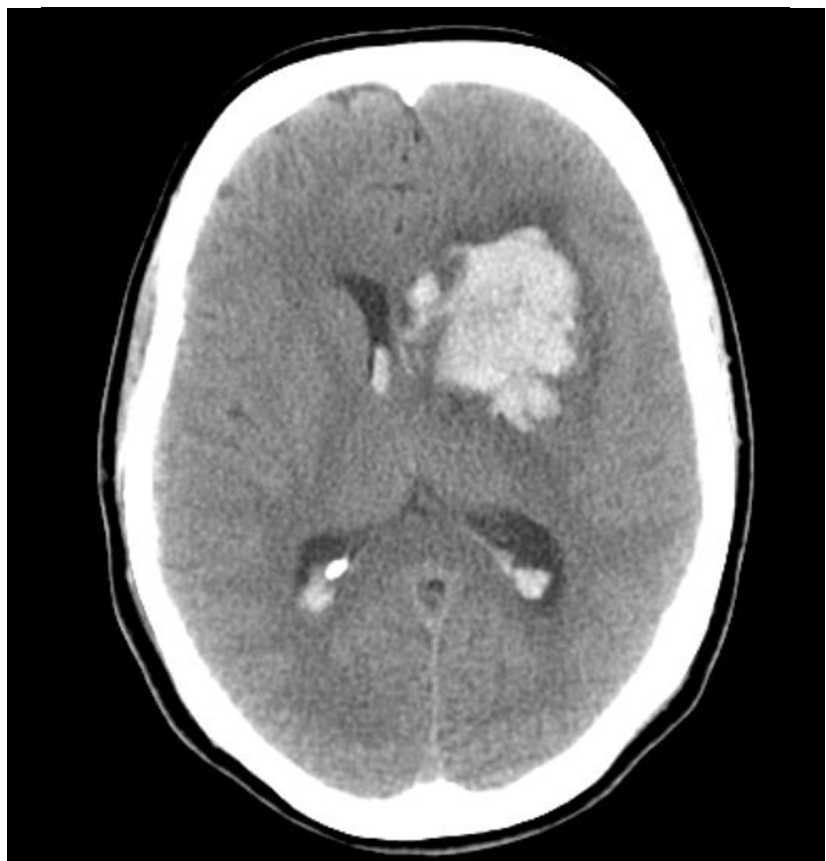

Figura 1 - TC cerebral simples demonstrando hematoma agudo estriato-capsular esquerdo com sinais de rutura para o sistema ventricular

anestesia epidural), sem intercorrências, inicia cefaleia occipital súbita, de intensidade crescente e que se associou a alteração da linguagem e diminuição de força nos membros direitos, motivo pelo qual foi levada ao serviço de urgência. À admissão apresentava afasia de Broca e hemiplegia direita. A tomografia computorizada (TC) então realizada revelou hematoma cerebral agudo estriato-capsular esquerdo (Fig.1), não se identificando alterações de relevo na angio-TC cerebral. Para despiste de lesões vasculares subjacentes foi realizada angiografia cerebral diagnóstica ao oitavo dia de internamento, identificando-se múltiplas estenoses segmentares envolvendo artérias intracranianas de grande e médio calibre, nomeadamente os segmentos terminais das artérias carótidas internas, ramos das artérias cerebrais médias, anteriores e posteriores, e das artérias basilar e vertebral esquerda (Fig. 2), alterações que no contexto clínico sugeriam SVCR. O estudo analítico realizado, incluindo estudo imunológico alargado e serologias, não demonstrou alterações valorizáveis. Para controlo do vasospasmo foi então iniciado tratamento com nimodipina (60 mg 4x/ dia, via oral) e hipervolémia controlada.

Ao $12^{\circ}$ dia de internamento apresentou crise parcial complexa com perda de contacto, versão cefálica para a esquerda e postura tónica do membro superior ipsilateral, com correspondente atividade paroxística ântero-lateral direita no electroencefalograma, tendo sido medicada com levetiracetam para controlo das crises e com prednisolona (30 mg/ dia, via oral) como complemento à terapêutica já instituída para controlo do vasospasmo. Realizou ressonância magnética (RM) encefálica ao $19^{\circ}$ dia de internamento que revelou enfarte agudo em território da artéria pericalosa direita (Fig. 3). O primeiro ecoDoppler transcraniano, efetuado nesta altura, demonstrou fluxo turbulento e de altas velocidades sistólica e diastólica nas artérias cerebrais médias (velocidade média de $130 \mathrm{~cm} / \mathrm{s}$ ) e na artéria cerebral anterior direita, não tendo sido possível identificar a artéria basilar. Neste contexto foi ponderada a realização de tratamento endovascular tendo-se realizado angio-TC cerebral (Fig. 4) que revelou marcada redução difusa do calibre das artérias cerebrais de grande, médio e pequeno calibre. Atendendo ao facto do vasospasmo ser severo e difuso, tornando a abordagem endovascular tecnicamente exigente e com maior risco de complicações, e ao facto de os défices neurológicos focais da doente serem secun-

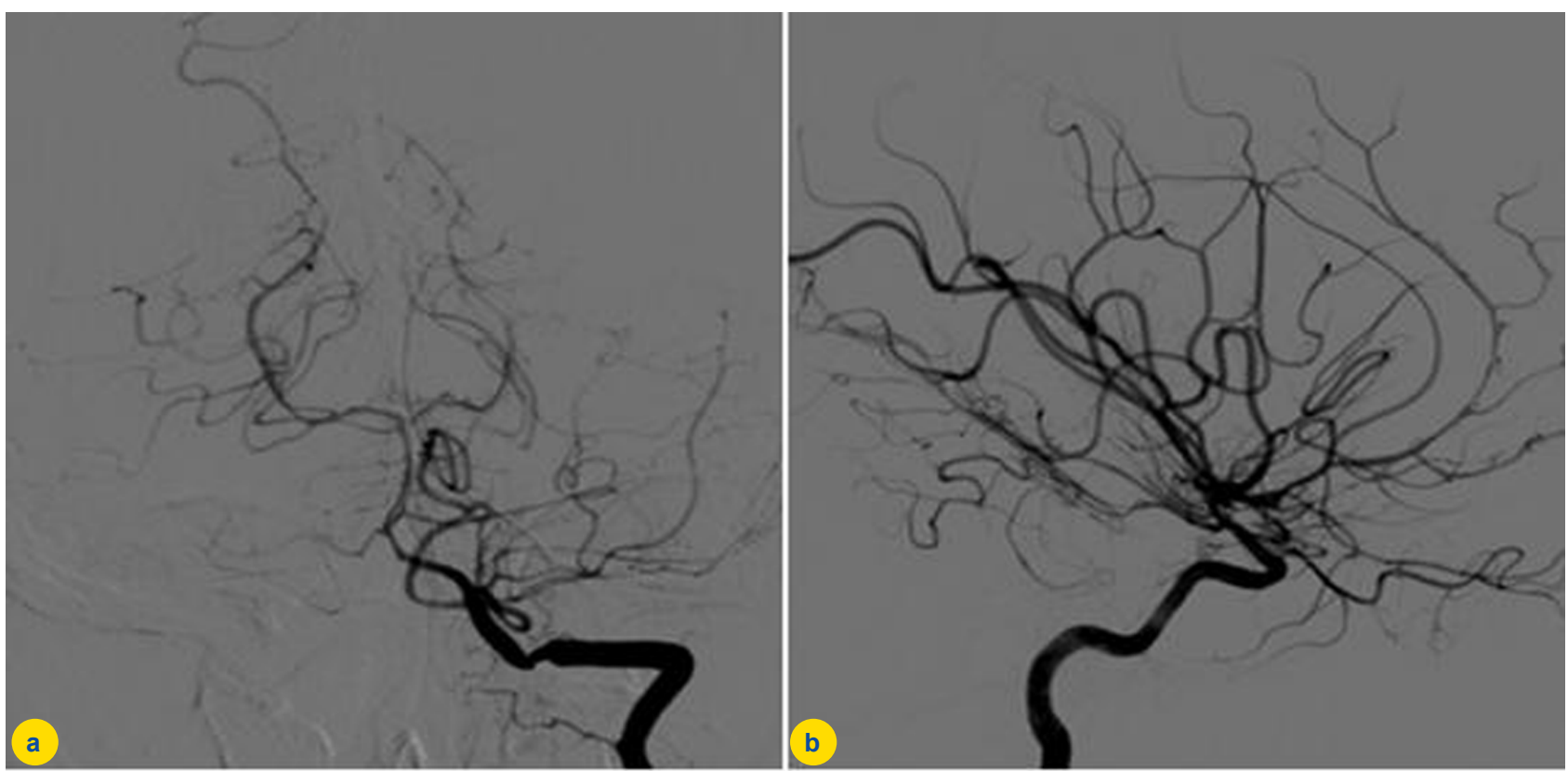

Figura 2 - Angiografia de subtração digital, imagens de face (a) e de perfil (b) após injeção nas artérias vertebral e carótida interna esquerdas, respetivamente. Estenoses segmentares no segmento terminal da artéria carótida interna e nas artérias caloso-marginal, vertebral, basilar e parieto-occipital direita. 

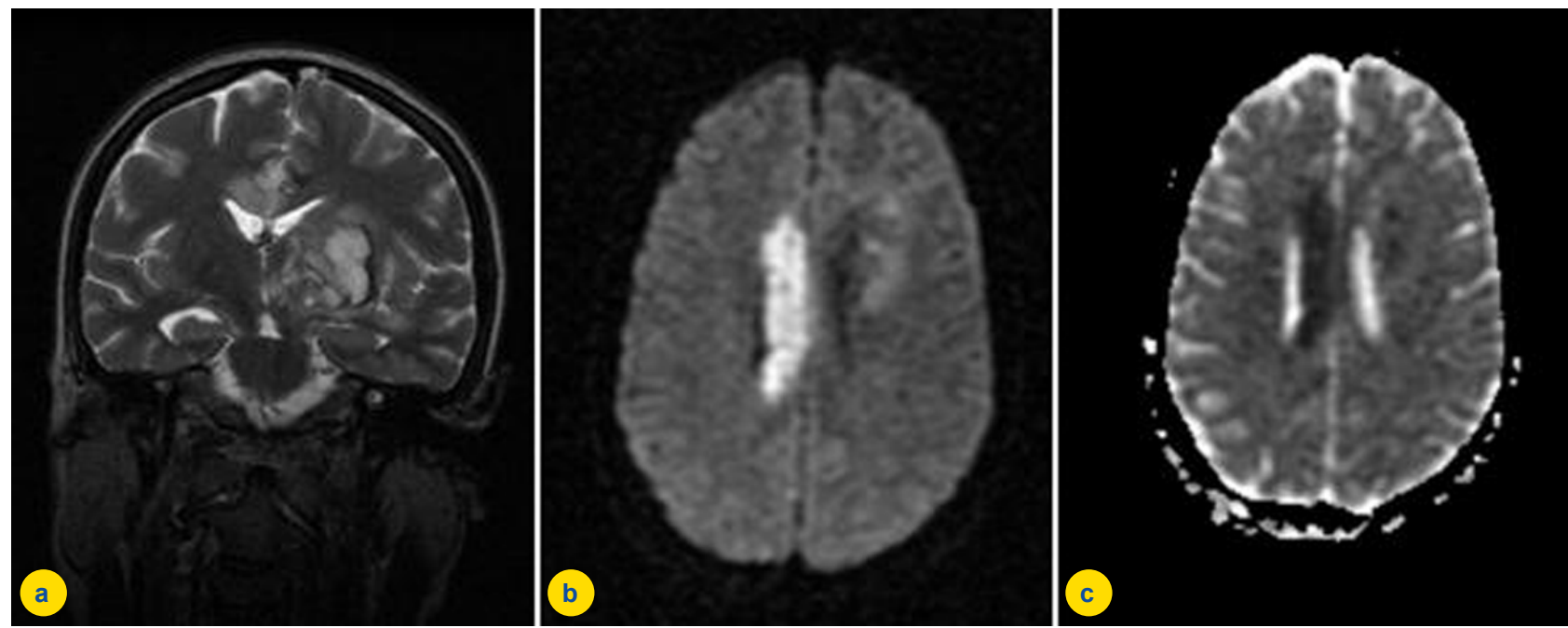

Figura 3 - RM encefálica, imagens coronais T2 (a) e axiais difusão (b) e mapa ADC (c). Lesão córtico-subcortical no corpo caloso e giro do cíngulo à direita com hipersinal em T2 e difusão e hipossinal no mapa ADC (restrição da difusão das moléculas de água), traduzindo enfarte recente em território da artéria pericalosa direita. Adicionalmente identifica-se hematoma subagudo estriato-capsular esquerdo.

dários a enfartes já estabelecidos, decidiu-se não avançar para o tratamento endovascular. Manteve-se, deste modo, a terapêutica médica em vigor, verificando-se melhoria do vasospasmo arterial no ecoDoppler transcraniano realizado ao $26^{\circ}$ dia de internamento e resolução do mesmo em novo estudo realizado dois dias depois.

A doente fez reabilitação fisiátrica em regime de internamento e aos sete meses após o início do quadro mantinha afasia de Broca moderada, mas com melhoria da hemiparesia direita (força muscular grau 4 no membro superior e grau 5 - no membro inferior). A angio-RM de controlo, realizada cinco meses após o início da clínica, demonstrou resolução do vasospasmo cerebral, persistindo apenas ligeira redução do calibre do topo da artéria carótida interna esquerda e porção inicial do segmento M1 da artéria cerebral média esquerda (Fig. 5).

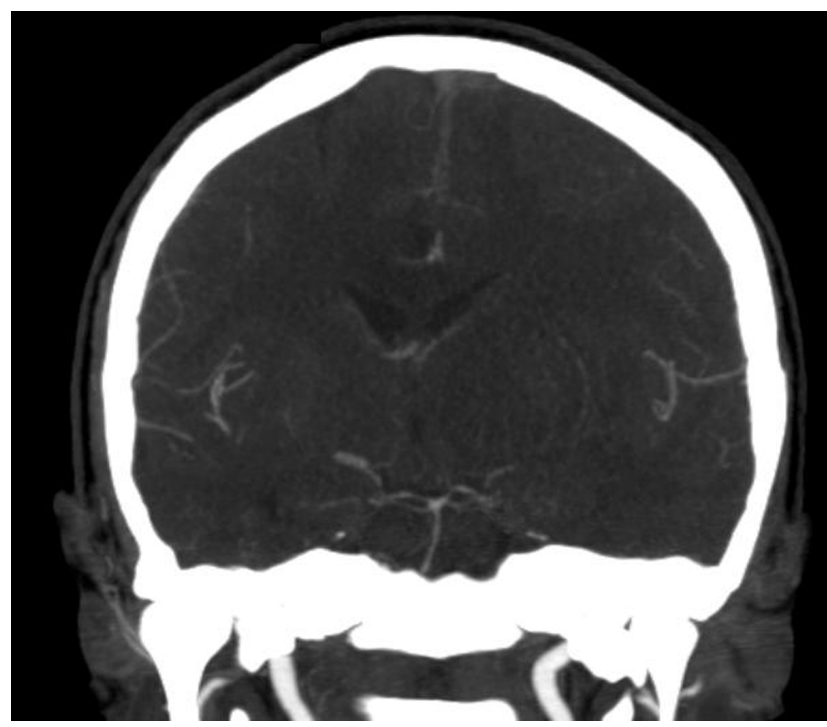

Figura 4 - Angio-TC cerebral, reformatações coronais. Estenose severa com preenchimento filiforme da artéria basilar.

\section{DISCUSSÃO}

Descrevemos um caso cuja apresentação clínica e achados angiográficos são altamente sugestivos de SVCR, hipótese sustentada pela resolução do vasospasmo cerebral no controlo angiográfico a médio prazo. Embora rara esta entidade é uma causa importante de acidentes vasculares cerebrais no puerpério, devendo ser considerada em puérperas que se apresentem com sintomas neurológicos agudos. ${ }^{5,7}$

Os exames complementares de imagem desempenham um papel fundamental para o seu diagnóstico, ao excluírem outras patologias mais frequentes e ao demonstrar a vasoconstrição segmentar das artérias cerebrais que caracteriza

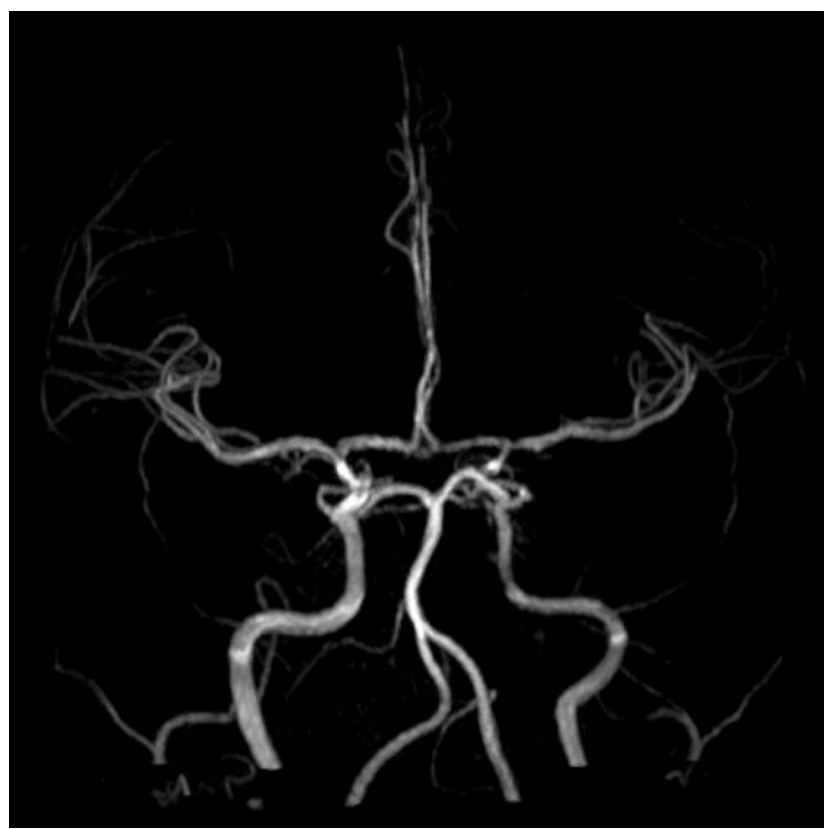

Figura 5 - Angio-RM por técnica 3D TOF, reformatações 3D. Resolução das estenoses arteriais intracranianas, persistindo apenas ligeira redução do calibre do topo da artéria carótida interna e segmento M1 da artéria cerebral média à esquerda. 
esta patologia. Neste capítulo, a angiografia cerebral clássica, embora sendo uma técnica invasiva, permanece o gold standard para o seu diagnóstico. ${ }^{2}$ Tal como aconteceu no nosso caso, o vasospasmo arterial pode não ser evidente no primeiro estudo angiográfico, 8,9 pelo que nos casos em que a suspeita clínica for elevada está indicada a repetição destes exames. ${ }^{4}$ Do ponto de vista imagiológico o diagnóstico diferencial faz-se principalmente com vasculite do sistema nervoso central, ${ }^{2}$ sendo que a resolução do vasospasmo cerebral no controlo angiográfico a médio prazo permite diferenciar estas duas entidades. ${ }^{2}$ Adicionalmente, as técnicas avançadas de RM que avaliam a parede arterial podem contribuir para este diagnóstico diferencial ainda na fase inicial da doença, dado que ao contrário do que ocorre nos processos vasculíticos, nas SVCR não foram observadas captações anómalas do produto de contraste na parede das artérias envolvidas. ${ }^{10} \mathrm{O}$ estudo laboratorial, incluindo estudo imunológico alargado, serologias e estudo do líquido cefalorraquidiano, é mais frequentemente normal na SVCR, devendo contudo ser realizado pela sua potencial utilidade na exclusão de outras patologias. ${ }^{1}$

Embora classicamente considerada uma patologia com prognóstico favorável, à semelhança do nosso caso o vasospasmo pode ser progressivo levando a uma evolução clínica desfavorável (Fig. 4).,7 É deste modo importante a vigilância clínica para detetar eventuais deteriorações neurológicas secundárias ${ }^{4,7}$ e um controlo imagiológico da vasoconstrição arterial, capítulo onde o ecoDoppler transcraniano, como método não invasivo, pode desempenhar um papel importante. ${ }^{6} \mathrm{Na}$ maior parte dos casos verifica-se resolução total do vasospasmo em três meses, contudo, tal como neste caso, alguns doentes podem apresentar cursos mais prolongados, devendo considerar-se haver 'resolução do vasospasmo' caso haja melhoria significativa do mesmo aos três meses (Fig. 5). ${ }^{2}$

O tratamento desta entidade ainda não está devidamente estabelecido e, atendendo ao facto do vasospasmo ser geralmente auto-limitado, não se sabe se alterará a história natural da doença. ${ }^{2,4}$ As opções terapêuticas mais frequentemente utilizadas são a administração de bloqueadores de canais de cálcio, de corticosteroides e de sulfato de magnésio, ${ }^{2,4}$ e a hipervolémia. ${ }^{6}$ Nos quadros refratários, o tratamento endovascular deve ser ponderado, ${ }^{4,6} \mathrm{com}$ administração intra-arterial de bloqueadores de canais de cálcio e/ou angioplastia com balão. Este tratamento só deve contudo ser realizado na ausência de resposta à melhor terapêutica médica e na presença de défices neurológicos focais em território de artérias envolvidas pelo vasospasmo e que não sejam secundários a enfartes já estabelecidos. ${ }^{11}$

\section{CONCLUSÃO}

Embora rara a SVCR é uma causa importante de acidentes vasculares cerebrais no puerpério, podendo apresentar cursos desfavoráveis com rápida progressão do vasospasmo arterial e, consequentemente, elevada morbilidade e mortalidade. É deste modo fundamental uma vigilância clínica e imagiológica intensa para deteção precoce de eventuais deteriorações secundárias.

\section{CONFLITOS DE INTERESSE}

Os autores declaram não existirem qualquer conflitos de interesse.

\section{FONTE DE FINANCIAMENTO}

Não foram atribuídas bolsas/subsídios para a realização do trabalho.

\section{REFERÊNCIAS}

1. Singhal $A B$, Bernstein RA. Postpartum angiopathy and other cerebral vasoconstriction syndromes. Neurocrit Care. 2005;3:91-7.

2. Chen SP, Fuh JL, Wang SJ. Reversible cerebral vasoconstriction syndrome: current and future perspectives. Expert Rev Neurother. 2011;11:1265-76.

3. Ducros A. Reversible cerebral vasoconstriction syndrome. Lancet Neurol. 2012;11:906-17

4. Fugate JE, Ameriso SF, Ortiz G, Schottlaender LV, Wijdicks EFM, Flemming KD, et al. Variable presentations of postpartum angiopathy. Stroke. 2012;43:670-6

5. Konstantinopoulos PA, Mousa S, Khairallah R, Mtanos G. Postpartum cerebral angiopathy: an importante diagnostic consideration in the postpartum period. Am J Obstet Gynecol. 2004;191:375-7.

6. Zak IT, Dulai HS, Kish K. Imaging of neurological disorders associated with pregnancy and the postpartum period. Radiographics. 2007;27:95108.
7. Fugate JE, Wijdicks EF, Parisi JE, Kallmes DF, Cloft HJ, Flemming KD, et al. Fulminant postpartum cerebral vasoconstriction syndrome. Arch Neurol. 2012;69:111-7.

8. Chen SP, Fuh JL, Wang SJ, Chang FC, Lirng JF, Fang YC, et al. Magnetic resonance angiography in reversible cerebral vasoconstriction syndromes. Ann Neurol. 2010;67:648-56.

9. Ducros A, Boukobza M, Porcher R, Sarov M, Valade D, Bousser $M G$. The clinical and radiological spectrum of reversible cerebral vasoconstriction syndrome: a prospective series of 67 patients. Brain. 2007;130:3091-101.

10. Mandell DM, Matouk CC, Farb RI, Krings T, Agid R, terBrugge K, et al. Vessel wall MRI to differentiate between reversible cerebral vasoconstriction syndrome and central nervous system vasculitis: preliminary results. Stroke. 2012;43:860-2.

11. Krings T, Geibprasert S, Ter Brugge KG. Case-based interventional neuroradiology. New York: Thieme; 2011. 


\section{Síndrome de Vasoconstrição Cerebral Reversível: uma Causa Importante de Acidentes Vasculares Cerebrais no Puerpério Acta Med Port 2014:27:515-518}

Publicado pela Acta Médica Portuguesa, a Revista Científica da Ordem dos Médicos

Av. Almirante Gago Coutinho, 151

1749-084 Lisboa, Portugal.

Tel: +351218428 215

E-mail: submissao@actamedicaportuguesa.com

www.actamedicaportuguesa.com

ISSN:0870-399X | e-ISSN: 1646-0758

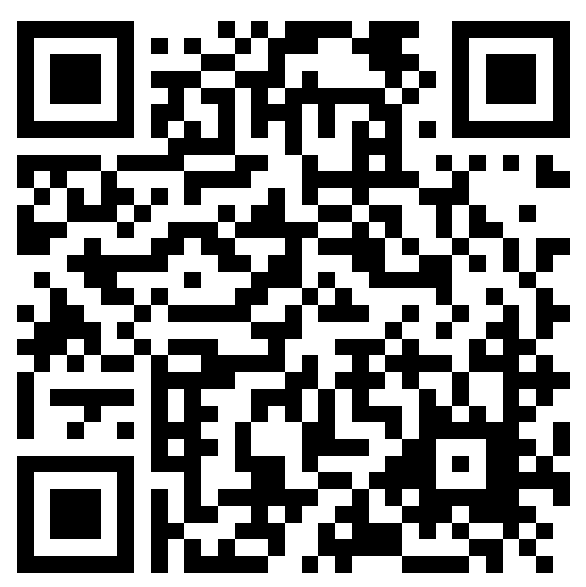

